\title{
On the irrationality of factorial series
}

\author{
by \\ Jaroslav Hančl (Ostrava) and Robert TiJdeman (Leiden)
}

1. Introduction. Let $\left(b_{n}\right)_{n=1}^{\infty}$ be a sequence of integers. In the present paper we study the irrationality of $R:=\sum_{n=1}^{\infty} b_{n} / n$ ! and, more generally, of

$$
R^{*}:=\sum_{N=1}^{\infty} \frac{b_{N}}{\prod_{n=1}^{N}(a n+b)}
$$

where $a$ and $b$ are given positive integers. In 1761 Lambert [10] proved the irrationality of $e=1+\sum_{n=1}^{\infty} 1 / n$ !. In 1873 Hermite [9] established the transcendence of $e$, which implies the irrationality of $\sum_{n=1}^{\infty} m^{n} / n$ ! for any nonzero integer $m$. In $1869 \mathrm{G}$. Cantor [2] showed that if $0 \leq b_{n}<n$, then $R$ is irrational if and only if $b_{n}>0$ infinitely often and $b_{n}<n-1$ infinitely often. On the other hand, if $b_{n} /(n-1)$ is constant for $n$ larger than some $n_{0}$, then $R \in \mathbb{Q}$. This is an exceptional case in many results.

Oppenheim [12] showed that both the condition of $b_{n}>0$ and the condition of $b_{n}<n-1$ can be relaxed. For example, it follows from his results that if $\left|b_{n}\right|<n$ for every $n$, then $R$ is rational if and only if $b_{n} /(n-1)$ is ultimately a fixed integer. Thus if $\left|b_{n}\right|<n-1$ for every $n$ and $R \in \mathbb{Q}$, then $b_{n}$ is ultimately equal to 0 . The results of Oppenheim were extended by the authors [8] who showed that if $n \nmid b_{n}$ for all $n, b_{n}=o\left(n^{2}\right)$ and $\liminf _{n \rightarrow \infty}\left|b_{n}\right| / n=0$, then $R$ is irrational. They further proved that $R$ is irrational if $\left(b_{n}\right)_{n=1}^{\infty}$ is a monotonic sequence of positive integers such that $b_{n}=O\left(n^{2}\right)$ and $\operatorname{gcd}\left(b_{n}, n-1\right)=o\left(b_{n}\right)$. Tijdeman and Yuan [14] extended another result of Oppenheim by showing that $R$ is irrational if $b_{n}=O(n)$ and the sequence $\left(b_{n} / n\right)_{n=1}^{\infty}$ has an irrational limit point. See also Hančl [6] and [7].

Erdős and Straus [5] started a series of results in which the size of the difference $b_{n+1}-b_{n}$ is a relevant factor. They used such results to establish the

2000 Mathematics Subject Classification: Primary 11J72. series.

Key words and phrases: irrationality, linear independence, infinite series, factorial

The first-named author is supported by grants 201/04/0381 and MSM6198898701. 
irrationality of $R$ in case $\left(b_{n}\right)_{n=1}^{\infty}$ represents a multiplicative or other arithmetic function. It follows from their result that if $b_{n}>0$ for all $n, b_{n+1}-b_{n}=$ $o(n)$ and $\liminf _{n \rightarrow \infty} n / b_{n}=0$, then $R$ is irrational. The authors [8] showed that the condition $\liminf \operatorname{in}_{n \rightarrow \infty} n / b_{n}=0$ can be replaced with the necessary condition that $b_{n} /(n-1)$ is not ultimately constant. Tijdeman and Yuan [14] showed that, moreover, the condition $b_{n}>0$ for all $n$ can be dropped: if $b_{n+1}-b_{n}=o(n)$, then $R \in \mathbb{Q}$ if and only if $b_{n} /(n-1)$ is ultimately a fixed integer. These results generalize Erdös' result [3] that $\sum_{n=1}^{\infty} p_{n} / n ! \notin \mathbb{Q}$, where $\left\{p_{n}\right\}_{n=1}^{\infty}$ is the sequence of consecutive prime numbers.

In fact Erdős claimed the irrationality of $\sum_{n=1}^{\infty} p_{n}^{k} / n ! \notin \mathbb{Q}$ for $k=$ $1,2, \ldots$, but unfortunately he proved only the case $k=1$. Oppenheim [12] showed that $\sum_{n=1}^{\infty} \varepsilon_{n} d_{n} / n !, \sum_{n=1}^{\infty} \varepsilon_{n} \sigma_{n} / n$ ! and $\sum_{n=1}^{\infty} \varepsilon_{n} \phi_{n} / n$ ! are irrational for all choices of $\varepsilon_{n} \in\{-1,1\}$, where $d(n), \sigma(n), \phi(n)$ denote the number of divisors, the sum of divisors, and the Euler function of $n$, respectively. A special case was treated by Erdős and Kac [4]. Erdős and Straus [5] proved that the numbers $1, \sum_{n=1}^{\infty} \sigma_{n} / n$ !, $\sum_{n=1}^{\infty} \phi_{n} / n$ ! and $\sum_{n=1}^{\infty} b_{n} / n$ !, where $\left|b_{n}\right|<n^{1 / 2-\varepsilon}$ for all large $n$ and $b_{n} \neq 0$ infinitely often, are linearly independent over the rationals. Most of the results mentioned were stated in greater generality in the original papers than above.

Tijdeman and Yuan [14] started to compare second order differences (cf. the proof of their Theorem 4.3). In the present paper we pursue this idea by studying $K$ th order differences. For doing so we have to impose stronger regularity conditions on the numbers $b_{n}$. Nevertheless the results are valid for a wide class of sequences $\left(b_{n}\right)_{n=1}^{\infty}$. Corollary 3.1 precisely states for which polynomials $P(x)$ with integer coefficients $\sum_{n=1}^{\infty} P(n) / n$ ! is rational. Section 3 further provides a method to establish the irrationality of a large class of numbers

$$
\sum_{N=1}^{\infty} \frac{f(N)}{\prod_{n=1}^{N}(a n+b)}
$$

where $f(N)$ is an integer-valued function satisfying $f(N)=(a N+b) F(N)$ $+O(1)$ and $F$ is a smooth function which does not grow faster than a polynomial. In particular it yields the irrationality of the following numbers:

$$
\sum_{n=1}^{\infty} \frac{\left[n^{\alpha}\right]}{n !}(\alpha \geq 0), \quad \sum_{n=1}^{\infty} \frac{\left[\log ^{\beta} n\right]}{n !}(\beta>0), \quad \sum_{n=1}^{\infty} \frac{\left[\exp \left(\log ^{\gamma} n\right)\right]}{n !}(0<\gamma<1) .
$$

In Section 4 the linear independence over the rationals of such numbers is treated. For example, linear independence is shown for the numbers

$$
1, \quad e, \quad \sum_{n=1}^{\infty} \frac{\left[n^{\alpha}\right]}{n !} \text { for all } \alpha \in \mathbb{R}_{+}, \alpha \notin \mathbb{Z} .
$$

The results remind us of the result by Loxton and van der Poorten [11] 
who proved by Mahler's method that $\sum_{n=1}^{\infty}[n \alpha] \beta^{n}$ is transcendental for $\alpha$ irrational and $\beta$ algebraic with $0<|\beta|<1$.

2. Basic lemmas. Let $a>0$ and $b$ be integers such that $a n+b \neq 0$ for every positive integer $n$. Let $\left\{b_{n}\right\}_{n=1}^{\infty}$ be a sequence of integers. We investigate under what conditions

$$
R^{*}=\sum_{N=1}^{\infty} \frac{b_{N}}{\prod_{n=1}^{N}(a n+b)}
$$

is irrational. Since all terms are rational, we may neglect the terms with $a n+b<0$ and assume without loss of generality that $b \geq 0$.

The following lemma dealing with the sum

$$
R_{N}^{*}:=\sum_{m=N}^{\infty} \frac{b_{m}}{\prod_{n=N}^{m}(a n+b)}
$$

is crucial. We denote the set of positive integers by $\mathbb{N}$.

Lemma 2.1. If $R^{*}=p / q$ for some $p \in \mathbb{Z}, q \in \mathbb{N}$, then $q R_{N}^{*} \in \mathbb{Z}$ for all $N$.

Proof. We have

$$
p \prod_{n=1}^{N-1}(a n+b)=q \sum_{m=1}^{N-1} b_{m} \prod_{n=m+1}^{N-1}(a n+b)+q \sum_{m=N}^{\infty} \frac{b_{m}}{\prod_{n=N}^{\infty}(a n+b)}
$$

and the first two terms are integers.

REMARK. If $q$ divides $\prod_{n=1}^{N-1}(a n+b)$, then we need not multiply by $q$ to obtain integers and can conclude that $R_{N}^{*}$ itself is an integer. If $q$ is coprime to $a$, this is the case for sufficiently large $N$. In particular, it is the case if $a=1$, hence for sequences $\sum_{n=1}^{\infty} b_{n} / n !$.

The following consequence of a theorem of Oppenheim implies that $R^{*}$ is irrational if $b_{n}=o(n)$, but not ultimately constant 0 .

Lemma 2.2 (Oppenheim [12, Theorem 8]). If $\left|b_{n}\right|<a n+b$ for all $n>n_{0}$ and $\liminf \operatorname{in}_{n \rightarrow \infty}\left|b_{n}\right| / n=0$, then $R$ is rational if and only if $b_{n}=0$ for all $n>n_{0}$.

The next lemma displays some well known properties of Stirling numbers of the second kind.

Lemma 2.3. Let $K$ be a nonnegative integer. Put

$$
S(r, K)=\frac{1}{K !} \sum_{j=0}^{K}(-1)^{K-j}\left(\begin{array}{c}
K \\
j
\end{array}\right) j^{r} .
$$

Then $S(r, K)=0$ if $r<K, S(r, K)=1$ if $r=K$ and $S(r, K) \in \mathbb{N}$ if $r>K>0$.

For a proof see [1, Section III.2]. 
The following lemma gives partial fractions of the denominator of $R^{*}$.

Lemma 2.4. Let $a>0, b, N$ and $s \geq 0$ be integers such that $a(N+i)+b$ $\neq 0$ for $i=0,1, \ldots, s$. Then

$$
\frac{1}{\prod_{i=0}^{s}(a(N+i)+b)}=\frac{1}{s ! a^{s}} \sum_{i=0}^{s}(-1)^{i}\left(\begin{array}{c}
s \\
i
\end{array}\right) \frac{1}{a(N+i)+b} .
$$

Proof. We prove the identity by induction on $s$. For $s=0$ identity (3) is trivial. Suppose that it holds for $s=n$. Then

$$
\begin{aligned}
& \frac{1}{\prod_{i=0}^{n+1}(a(N+i)+b)} \\
&=\frac{1}{(n+1) a}\left(\frac{1}{\prod_{i=0}^{n}(a(N+i)+b)}-\frac{1}{\prod_{i=1}^{n+1}(a(N+i)+b)}\right) \\
&=\frac{1}{(n+1) ! a^{n+1}}\left(\sum_{i=0}^{n}(-1)^{i}\left(\begin{array}{c}
n \\
i
\end{array}\right) \frac{1}{a(N+i)+b}\right. \\
&=\frac{\left.-\sum_{i=1}^{n+1}(-1)^{i-1}\left(\begin{array}{c}
n \\
i-1
\end{array}\right) \frac{1}{a(N+i)+b}\right)}{(n+1) ! a^{n+1}}\left(\begin{array}{c}
1 \\
a N+b
\end{array}+\frac{(-1)^{n+1}}{a(N+n+1)+b}\right. \\
&\left.+\sum_{i=1}^{n}(-1)^{i}\left(\left(\begin{array}{c}
n \\
i
\end{array}\right)+\left(\begin{array}{c}
n \\
i-1
\end{array}\right)\right) \frac{1}{a(N+i)+b}\right) \\
&= \frac{1}{(n+1) ! a^{n+1}} \sum_{i=0}^{n+1}(-1)^{i}\left(\begin{array}{c}
n+1 \\
i
\end{array}\right) \frac{1}{a(N+i)+b} .
\end{aligned}
$$

This completes the induction step.

The last lemma of the section will be used for all theorems except for Theorem 3.1 and Theorem 3.2. We use the convention that an empty product equals 1 .

Lemma 2.5. Let $K \geq 0, a>0$ and $b$ be given integers such that $a n+b \neq 0$ for every $n \in \mathbb{N}$. Let $H: \mathbb{R} \rightarrow \mathbb{R}_{+}$be a $K$ times continuously differentiable function such that $H(x) \neq 0$ for $x>x_{0}$. Suppose we have

$$
\begin{aligned}
& H(N+j)=\sum_{r=0}^{K} \frac{H^{(r)}(N)}{r !} j^{r}+O\left(\frac{H(N)}{N^{K+1}}\right) \\
& \qquad \text { for } j=0,1, \ldots, K \text { as } N \rightarrow \infty
\end{aligned}
$$


and

$$
H^{(r)}(N)=O\left(\frac{H(N)}{N^{r}}\right) \quad \text { for } r=0,1, \ldots, K \text { as } N \rightarrow \infty
$$

Put

$$
R_{K}^{*}(N)=\sum_{j=0}^{K}(-1)^{j}\left(\begin{array}{c}
K \\
j
\end{array}\right) \sum_{s=0}^{\infty} \frac{H(N+j+s)}{\prod_{n=N+j}^{N+j+s-1}(a n+b)} .
$$

Then

$$
R_{K}^{*}(N)=(-1)^{K} H^{(K)}(N)+O\left(H(N) N^{-K-1}\right) \quad \text { as } N \rightarrow \infty .
$$

Proof. Let $N$ be sufficiently large. Note that for $j=0,1, \ldots, K$, by (4) and (5),

$$
\begin{aligned}
H(N+j) & =O\left(H(N) \sum_{r=0}^{K+1} \frac{1}{r !}\left(\frac{j}{N}\right)^{r}\right)=O\left(H(N) e^{j / N}\right) \\
& =O(H(N)) \quad \text { as } N \rightarrow \infty
\end{aligned}
$$

Write

$$
R_{K}^{*}(N)=R_{K, 1}^{*}(N)+R_{K, 2}^{*}(N)+R_{K, 3}^{*}(N)
$$

where

$$
\begin{aligned}
& R_{K, 1}^{*}(N)=\sum_{j=0}^{K}(-1)^{j}\left(\begin{array}{c}
K \\
j
\end{array}\right) H(N+j), \\
& R_{K, 2}^{*}(N)=\sum_{j=0}^{K}(-1)^{j}\left(\begin{array}{c}
K \\
j
\end{array}\right) \sum_{s=1}^{K} \frac{H(N+j+s)}{\prod_{n=N+j}^{N+j+s-1}(a n+b)}, \\
& R_{K, 3}^{*}(N)=\sum_{j=0}^{K}(-1)^{j}\left(\begin{array}{c}
K \\
j
\end{array}\right) \sum_{s=K+1}^{\infty} \frac{H(N+j+s)}{\prod_{n=N+j}^{N+j+s-1}(a n+b)} .
\end{aligned}
$$

By Lemma 2.3 and (4) we have

$$
\begin{aligned}
R_{K, 1}^{*}(N) & =\sum_{j=0}^{K}(-1)^{j}\left(\begin{array}{c}
K \\
j
\end{array}\right) \sum_{r=0}^{K} \frac{H^{(r)}(N)}{r !} j^{r}+O\left(H(N) N^{-K-1}\right) \\
& =\sum_{r=0}^{K} \frac{H^{(r)}(N)}{r !} \sum_{j=0}^{K}(-1)^{j}\left(\begin{array}{c}
K \\
j
\end{array}\right) j^{r}+O\left(H(N) N^{-K-1}\right) \\
& =(-1)^{K} H^{(K)}(N)+O\left(H(N) N^{-K-1}\right) \quad \text { as } N \rightarrow \infty .
\end{aligned}
$$

We now turn to $R_{K, 2}^{*}(N)$. By Lemma 2.4, (4), (7), Lemma 2.3, and (5), we have 


$$
\begin{aligned}
& R_{K, 2}^{*}(N)=\sum_{j=0}^{K}(-1)^{j}\left(\begin{array}{c}
K \\
j
\end{array}\right) \sum_{s=1}^{K} \frac{H(N+s+j)}{(s-1) ! a^{s-1}} \\
& \times \sum_{i=0}^{s-1}(-1)^{i}\left(\begin{array}{c}
s-1 \\
i
\end{array}\right) \frac{1}{a(N+i+j)+b} \\
& =\sum_{j=0}^{K}(-1)^{j}\left(\begin{array}{c}
K \\
j
\end{array}\right) \sum_{s=1}^{K} \sum_{r=0}^{K} \frac{H^{(r)}(N+s) j^{r}}{r !(s-1) ! a^{s-1}} \\
& \times \sum_{i=0}^{s-1}(-1)^{i}\left(\begin{array}{c}
s-1 \\
i
\end{array}\right) \sum_{m=1}^{\infty} \frac{(-1)^{m-1}(a j)^{m-1}}{(a(N+i)+b)^{m}}+O\left(\sum_{s=1}^{K} \frac{H(N+s)}{(N+s)^{K+1}}\right) \\
& =\sum_{s=1}^{K} \sum_{r=0}^{K} \frac{H^{(r)}(N+s)}{r !(s-1) ! a^{s-1}} \sum_{i=0}^{s-1}(-1)^{i}\left(\begin{array}{c}
s-1 \\
i
\end{array}\right) \\
& \times \sum_{m=1}^{\infty} \frac{(-a)^{m-1}}{(a(N+i)+b)^{m}} \sum_{j=0}^{K}(-1)^{j}\left(\begin{array}{c}
K \\
j
\end{array}\right) j^{r+m-1}+O\left(\sum_{s=1}^{K} \frac{H(N)}{N^{K+1}}\right) \\
& =\sum_{s=1}^{K} \sum_{r=0}^{K} \frac{H^{(r)}(N+s)}{r !(s-1) ! a^{s-1}} \sum_{i=0}^{s-1}(-1)^{i}\left(\begin{array}{c}
s-1 \\
i
\end{array}\right) \\
& \times \sum_{m=K-r+1}^{\infty} \frac{(-a)^{m-1}}{(a(N+i)+b)^{m}} \sum_{j=0}^{K}(-1)^{j}\left(\begin{array}{c}
K \\
j
\end{array}\right) j^{r+m-1}+O\left(\frac{H(N)}{N^{K+1}}\right) \\
& =O\left(\sum_{r=0}^{K} \frac{H(N)}{N^{r}} \sum_{m=K-r+1}^{\infty}\left(\frac{K}{N}\right)^{m}\right)+O\left(\frac{H(N)}{N^{K+1}}\right) .
\end{aligned}
$$

Thus

$$
R_{K, 2}^{*}(N)=O\left(\frac{H(N)}{N^{K+1}}\right) .
$$

Finally we estimate $R_{K, 3}^{*}(N)$. By $(7)$ there exists a constant $c>1$ such that $H(n+1)<c H(n)$ for all sufficiently large $n$. Hence $H(N+s)<c^{s} H(N)$ for every positive integer $s$. It follows that

$$
R_{K, 3}^{*}(N)=O\left(\sum_{s=K+1}^{\infty} \frac{H(N+s)}{\prod_{n=N}^{N+s-1}(a n+b)}\right)=O\left(\sum_{s=K+1}^{\infty} \frac{H(N) c^{s}}{N^{s}}\right)
$$

Hence

$$
R_{K, 3}^{*}(N)=O\left(\frac{H(N)}{N^{K+1}}\right) \quad \text { as } N \rightarrow \infty .
$$

The combination of (8), (9), (10) and (11) yields (6).

REMARK. In applications of Lemma 2.5 the integer $K$ is usually chosen as the smallest nonnegative integer such that $H^{(K)}(N) \rightarrow 0$ as $N \rightarrow \infty$. 
3. Irrationality. The next lemma implies that $\sum_{n=1}^{\infty} P(n) / n$ ! for $P(x)$ $\in \mathbb{Q}[x]$ is a linear combination over $\mathbb{Q}$ of 1 and $e$.

Lemma 3.1. Let $a>0$ and $b$ be integers such that $a n+b \neq 0$ for every $n \in \mathbb{N}$. Suppose that $P(x)=\sum_{i=0}^{T} a_{i} x^{i}$ is a polynomial with rational coefficients. Let $d$ be the least common denominator of $a_{0}, a_{1}, \ldots, a_{T}$. Then there exist rational numbers $Q_{0}$ and $Q_{1}$ such that

$$
\sum_{N=1}^{\infty} \frac{P(N)}{\prod_{n=1}^{N}(a n+b)}=Q_{0}+Q_{1} \sum_{N=1}^{\infty} \frac{1}{\prod_{n=1}^{N}(a n+b)}
$$

where

$$
\begin{aligned}
& a^{T} d Q_{0} \\
& =\sum_{i=0}^{T} a_{i} \sum_{k=0}^{i} \sum_{h=k}^{i}\left(\begin{array}{l}
i \\
h
\end{array}\right) a^{T-i+h-k}(-b)^{i-h} S(h, k) \sum_{N=1}^{k} \prod_{n=0}^{k-N-1}(b-n a)
\end{aligned}
$$

and

$$
a^{T} d Q_{1}=\sum_{i=0}^{T} a_{i} \sum_{k=0}^{i} \sum_{h=k}^{i}\left(\begin{array}{l}
i \\
h
\end{array}\right) a^{T-i+h-k}(-b)^{i-h} S(h, k)
$$

are integers.

Proof. We can write

$$
x^{i}=\sum_{k=0}^{i} b_{i, k} \prod_{m=1}^{k}(a(x-m+1)+b) .
$$

On substituting $x=-b / a+r$ for $r=0,1, \ldots, i$ into equation (14) we get

$$
\begin{aligned}
\left(-\frac{b}{a}+r\right)^{i} & =\sum_{k=0}^{i} b_{i, k} \prod_{m=1}^{k}\left(a\left(-\frac{b}{a}+r-m+1\right)+b\right) \\
& =\sum_{k=0}^{i} b_{i, k} a^{k} \frac{r !}{(r-k) !} .
\end{aligned}
$$

We consider (15) as a system of $i+1$ equations with $i+1$ unknowns $b_{i, 0}, b_{i, 1}, \ldots, b_{i, i}$. Using the fact that $r \leq i$ we find that the solution of this system for $k=0,1, \ldots, i$ is given by

$$
b_{i, k}=\frac{1}{k ! a^{k}} \sum_{j=0}^{k}(-1)^{j}\left(\begin{array}{l}
k \\
j
\end{array}\right)\left(-\frac{b}{a}+k-j\right)^{i} .
$$

We write

$$
\sum_{N=1}^{\infty} \frac{P(N)}{\prod_{n=1}^{N}(a n+b)}=\sum_{i=0}^{T} a_{i} \sum_{N=1}^{\infty} \frac{N^{i}}{\prod_{n=1}^{N}(a n+b)} .
$$


From this, (14) and (16) we obtain

$$
\begin{aligned}
& \sum_{N=1}^{\infty} \frac{P(N)}{\prod_{n=1}^{N}(a n+b)}=\sum_{i=0}^{T} a_{i} \sum_{k=0}^{i} b_{i, k} \sum_{N=1}^{\infty} \frac{\prod_{m=1}^{k}(a(N-m+1)+b)}{\prod_{n=1}^{N}(a n+b)} \\
& =\sum_{i=0}^{T} a_{i} \sum_{k=0}^{i} b_{i, k} \sum_{N=1}^{k} \prod_{n=0}^{k-N-1}(b-n a)+\sum_{i=0}^{T} a_{i} \sum_{k=0}^{i} b_{i, k} \sum_{N=k+1}^{\infty} \frac{1}{\prod_{n=1}^{N-k}(a n+b)} \\
& =: Q_{0}+Q_{1} \sum_{N=1}^{\infty} \frac{1}{\prod_{n=1}^{N}(a n+b)}
\end{aligned}
$$

where $Q_{0}$ and $Q_{1}$ are rational numbers. We have, by (16) and Lemma 2.3,

$$
\begin{aligned}
b_{i, k} & =\frac{1}{k ! a^{k}} \sum_{j=0}^{k}(-1)^{j}\left(\begin{array}{c}
k \\
j
\end{array}\right) \sum_{h=0}^{i}\left(\begin{array}{c}
i \\
h
\end{array}\right)\left(-\frac{b}{a}\right)^{i-h}(k-j)^{h} \\
& =\frac{1}{k ! a^{k}} \sum_{h=0}^{i}\left(\begin{array}{l}
i \\
h
\end{array}\right)\left(-\frac{b}{a}\right)^{i-h} \sum_{j=0}^{k}(-1)^{j}\left(\begin{array}{l}
k \\
j
\end{array}\right)(k-j)^{h} \\
& =\sum_{h=k}^{i}\left(\begin{array}{l}
i \\
h
\end{array}\right) \frac{1}{a^{k}}\left(-\frac{b}{a}\right)^{i-h} S(h, k)
\end{aligned}
$$

where $S(h, k)$ is an integer. Note that the exponent of $a$ in $\left(1 / a^{k}\right)(-b / a)^{i-h}$ has minimal value $-i$, viz. if $h=k$. Hence $a^{i} b_{i, k}$ is an integer for $i=$ $0,1, \ldots, k$. We deduce that $d a^{T} Q_{0}$ and $d a^{T} Q_{1}$ are the integers given by (12) and (13), respectively.

THEOREM 3.1. Let $a>0$ and $b$ be integers such that $a n+b \neq 0$ for every $n \in \mathbb{N}$. Let $P(x)=\sum_{i=0}^{T} a_{i} x^{i} \in \mathbb{Z}[x]$. Then

$$
R^{*}:=\sum_{N=1}^{\infty} \frac{P(N)}{\prod_{n=1}^{N}(a n+b)}
$$

is rational if and only if

$$
Q_{1}=\sum_{i=0}^{T} a_{i} \sum_{k=0}^{i} \frac{1}{k ! a^{k}} \sum_{j=0}^{k}(-1)^{j}\left(\begin{array}{l}
k \\
j
\end{array}\right)\left(-\frac{b}{a}+k-j\right)^{i}=0 .
$$

Proof. It is obvious that $R^{*}$ is absolutely convergent. It follows immediately from Lemma 3.1 that if (17) holds, then $R^{*} \in \mathbb{Q}$. On the other hand, suppose (17) does not hold. By Oppenheim's theorem (Lemma 2.2) we know that

$$
\sum_{N=1}^{\infty} \frac{1}{\prod_{n=1}^{N}(a n+b)}
$$

is irrational. Hence, by Lemma $3.1, R^{*}$ is irrational. 
Corollary 3.1. Let $P(x)=\sum_{i=0}^{T} a_{i} x^{i} \in \mathbb{Z}[x]$. Then $\sum_{N=1}^{\infty} P(N) / N$ ! is rational if and only if $\sum_{i=0}^{T} a_{i} \sum_{k=0}^{i} S(i, k)=0$. If $a_{T}>0$ and $a_{i} \geq 0$ for $i=0,1, \ldots, T-1$, then $\sum_{N=1}^{\infty} P(N) / N ! \notin \mathbb{Q}$.

Proof. Apply Theorem 3.1 with $a=1, b=0$, and Lemma 2.3.

REMARK. We recall that $\sum_{n=1}^{\infty}(n-1) / n !=1 \in \mathbb{Q}$. Hence the condition on the nonnegativity of the coefficients in the second statement cannot be dropped.

Corollary 3.2. Let $a>0$ and $b$ be integers such that $a n+b \neq 0$ for every $n \in \mathbb{N}$. Let $P(x)=\sum_{i=0}^{T} a_{i} x^{i} \in \mathbb{Z}[x]$. If $a \nmid a_{T}(b-1)^{T}$ then $R^{*}$ is irrational.

Proof. Observe that, by (13) and Lemma 2.3, the terms in $a^{T} Q_{1}$ are divisible by $a$ unless $h=k, i=T$. Hence, by Lemma 2.3 again,

$$
a^{T} Q_{1} \equiv a_{T} \sum_{k=0}^{T}\left(\begin{array}{l}
T \\
k
\end{array}\right)(-b)^{T-k} S(k, k)=a_{T}(1-b)^{T} \bmod a .
$$

TheOREM 3.2. Let $a>0$ and $b$ be integers such that $a n+b \neq 0$ for every $n \in \mathbb{N}$. Let $P(x)=\sum_{i=0}^{T} a_{i} x^{i} \in \mathbb{Q}[x]$. Let $f: \mathbb{N} \rightarrow \mathbb{Z}$ be a sequence such that $f(N)=P(N)+o(N)$ as $N \rightarrow \infty$. Suppose

Then

$$
\sum_{N=1}^{\infty} \frac{f(N)}{\prod_{n=1}^{N}(a n+b)} \in \mathbb{Q}
$$

$$
f(N)=P(N)-Q_{1} \quad \text { for all } N
$$

where $Q_{1}$ is given by $(13)$.

Proof. We have, by Lemma 3.1,

$$
\begin{aligned}
\sum_{N=1}^{\infty} \frac{f(N)}{\prod_{n=1}^{N}(a n+b)} & =\sum_{N=1}^{\infty} \frac{P(N)}{\prod_{n=1}^{N}(a n+b)}+\sum_{N=1}^{\infty} \frac{f(N)-P(N)}{\prod_{n=1}^{N}(a n+b)} \\
& =Q_{0}+Q_{1} \sum_{N=1}^{\infty} \frac{1}{\prod_{n=1}^{N}(a n+b)}+\sum_{N=1}^{\infty} \frac{f(N)-P(N)}{\prod_{n=1}^{N}(a n+b)} \\
& =Q_{0}+\sum_{N=1}^{\infty} \frac{Q_{1}+(f(N)-P(N))}{\prod_{n=1}^{N}(a n+b)}
\end{aligned}
$$

The numerator of the last fraction is a rational number which is $o(N)$ as $N \rightarrow \infty$ and has a denominator which is independent of $N$. Hence, by Lemma 2.2, $Q_{1}+f(N)-P(N)=0$ for $N \geq N_{0}$. It follows that $f(N)=$ $P(N)-Q_{1}$.

Corollary 3.3. Under the conditions of Theorem 3.2 we have $P(N) \equiv$ $Q_{1} \bmod 1$ for all $N$ and therefore $d Q_{1} \in \mathbb{Z}$. 
THEOREM 3.3. Let $a>0$ and $b$ be integers such that $a n+b \neq 0$ for every $n \in \mathbb{N}$. Let $P(x)=\sum_{i=0}^{T} a_{i} x^{i} \in \mathbb{R}[x]$. Let $f: \mathbb{N} \rightarrow \mathbb{Z}$ be a sequence such that $f(N)=(a N+b) P(N)+O(1)$ as $N \rightarrow \infty$. Suppose

$$
\sum_{N=1}^{\infty} \frac{f(N)}{\prod_{n=1}^{N}(a n+b)} \in \mathbb{Q} .
$$

Then $a_{T}, a_{T-1}, \ldots, a_{1}, a_{0} \in \mathbb{Q}$.

Proof. Suppose

$$
\sum_{N=1}^{\infty} \frac{f(N)}{\prod_{n=1}^{N}(a n+b)}=\frac{p}{q}
$$

where $p$ and $q>0$ are coprime integers. Let $U$ be the largest index $i$ with $a_{i} \notin \mathbb{Q}$. Let $d$ be a common denominator of $a_{U+1}, \ldots, a_{T}$. Put $P_{1}(x)=$ $\sum_{i=0}^{U} a_{i} x^{i}$ and $P_{2}(x)=(a x+b) \sum_{i=U+1}^{T} a_{i} x^{i}$. Then we have, by Lemma 3.1,

$$
\begin{aligned}
a^{T} d p & =a^{T} d q \sum_{N=1}^{\infty} \frac{f(N)}{\prod_{n=1}^{N}(a n+b)}=q \sum_{N=1}^{\infty} \frac{a^{T} d\left(f(N)-P_{2}(N)+P_{2}(N)\right)}{\prod_{n=1}^{N}(a n+b)} \\
& =q a^{T} d Q_{2,0}+q \sum_{N=1}^{\infty} \frac{a^{T} d\left(f(N)-P_{2}(N)\right)+a^{T} d Q_{2,1}}{\prod_{n=1}^{N}(a n+b)}
\end{aligned}
$$

where $Q_{2,0}$ and $Q_{2,1}$ are rational numbers corresponding to $P_{2}$ according to Lemma 3.1. From this and Lemma 2.1 we deduce that for every positive integer $N$ the number

$$
R_{N}^{*}:=q a^{T} d \sum_{s=0}^{\infty} \frac{f(N+s)-P_{2}(N+s)+Q_{2,1}}{\prod_{n=N}^{N+s}(a n+b)}
$$

is an integer. From the definition of $P_{1}, P_{2}$ and the assumption on $f$ it follows that

$$
R_{N}^{*}=q a^{T} d \sum_{s=0}^{\infty} \frac{P_{1}(N+s)}{\prod_{n=N}^{N+s-1}(a n+b)}+O\left(\frac{1}{N}\right) .
$$

This combined with Lemma 2.5 applied to $H(X)=q a^{T} d P_{1}(X+j)$ implies that the number

$$
\begin{aligned}
R_{U}^{*}(N) & :=\sum_{j=0}^{U}(-1)^{j}\left(\begin{array}{c}
U \\
j
\end{array}\right) R_{N+j}^{*} \\
& =(-1)^{U} q a^{T} d P_{1}^{(U)}(N)+O\left(\frac{1}{N}\right)=(-1)^{U} q a^{T} U ! d a_{U}+O\left(\frac{1}{N}\right)
\end{aligned}
$$

is an integer. This is a contradiction for a sufficiently large number $N$.

Corollary 3.4. Let $P(x)=\sum_{i=0}^{T} a_{i} x^{i} \in \mathbb{R}[x]$ with nonnegative coefficients and $a_{T}>0$. If $\sum_{N=1}^{\infty}[P(N)] / N ! \in \mathbb{Q}$, then $T=0$ and $\left[a_{0}\right]=0$. 
Proof. Suppose $\sum_{N=1}^{\infty}[P(N)] / N ! \in \mathbb{Q}$. It follows from Theorem 3.3 with $[P(N)]=N\left(\sum_{i=1}^{T} a_{i} N^{i-1}\right)+\left(a_{0}+O(1)\right)$ that $a_{T}, a_{T-1}, \ldots, a_{1}$ are rational. Therefore we may assume without loss of generality that $a_{0} \in \mathbb{Q}$. Let $d$ be the common denominator of $a_{T}, a_{T-1}, \ldots, a_{0}$. By Theorem 3.2 applied with $f(N)=[P(N)]$ we obtain $P(N)-[P(N)]=Q_{1}$ for all $N$ where $Q_{1}$ is a rational number. Hence $Q_{1}$ equals $a_{0}-\left[a_{0}\right]$. It follows that $[P(x)]=$ $\sum_{i=1}^{T} a_{i} x^{i}+\left[a_{0}\right] \in \mathbb{Q}[x]$. Since $[P(x)]$ has nonnegative rational coefficients and $a_{T}>0$, we deduce by applying Corollary 3.1 to $d[P(x)]=\sum_{i=1}^{T} d a_{i} x^{i}+$ $d\left[a_{0}\right] \in \mathbb{Z}[x]$ that $T=0$ and $\left[a_{0}\right]=0$.

REMARK. The corollary implies that for any positive integer $K$ the sum $\sum_{n=1}^{\infty}\left[\beta n^{K}\right] / n$ ! is a strictly increasing function of $\beta>0$ which does not take rational values. This phenomenon will be met several times later on.

Theorem 3.4. Let $K \geq 0, a>0$ and $b$ be given integers such that $a n+b \neq 0$ for every $n \in \mathbb{N}$. Let $F: \mathbb{R}_{+} \rightarrow \mathbb{R}_{+}$be $a K+1$ times continuously differentiable function such that

$$
\begin{gathered}
F(N+j)=\sum_{r=0}^{K} \frac{F^{(r)}(N)}{r !} j^{r}+O\left(\frac{F(N)}{N^{K+1}}\right) \\
\quad \text { for } j=0,1, \ldots, K \text { as } N \rightarrow \infty, \\
F^{(r)}(N)=O\left(\frac{F(N)}{N^{r}}\right) \quad \text { for } r=0,1, \ldots, K \text { as } N \rightarrow \infty, \\
\lim _{N \rightarrow \infty} F^{(K)}(N)=0, \quad \lim _{N \rightarrow \infty} \frac{N^{K+1}\left|F^{(K)}(N)\right|}{F(N)}=\infty, \\
\limsup _{N \rightarrow \infty} N\left|F^{(K)}(N)\right|=\infty .
\end{gathered}
$$

Let $f: \mathbb{N} \rightarrow \mathbb{Z}$ be a sequence such that

$$
R^{*}:=\sum_{N=1}^{\infty} \frac{f(N)}{\prod_{n=1}^{N}(a n+b)}
$$

is absolutely convergent and $f(N)=(a N+b) F(N)+O(1)$ as $N \rightarrow \infty$. Then $R^{*}$ is irrational.

Proof. Let $N$ be sufficiently large. Suppose $R^{*}=p / q$ where $p$ and $q>0$ are coprime integers. Put

$$
R_{K}^{*}(N)=\sum_{j=0}^{K}(-1)^{j}\left(\begin{array}{c}
K \\
j
\end{array}\right) \sum_{s=0}^{\infty} \frac{f(N+j+s)}{\prod_{n=N+j}^{N+j+s}(a n+b)} .
$$


By Lemma 2.1, $q R_{K}^{*}(N)$ is an integer for every positive integer $N$. We have, by Lemma 2.5 ,

$$
\begin{aligned}
R_{K}^{*}(N) & =\sum_{j=0}^{K}(-1)^{j}\left(\begin{array}{c}
K \\
j
\end{array}\right) \sum_{s=0}^{\infty} \frac{F(N+j+s)}{\prod_{n=N+j}^{N+j+s-1}(a n+b)}+O\left(\frac{1}{N}\right) \\
& =(-1)^{K} F^{(K)}(N)+O\left(\frac{F(N)}{N^{K+1}}+\frac{1}{N}\right) \\
& =(-1)^{K} F^{(K)}(N)\left(1+O\left(\frac{F(N)}{N^{K+1}\left|F^{(K)}(N)\right|}\right)\right)+O\left(\frac{1}{N}\right) .
\end{aligned}
$$

By (20) the right-hand side tends to 0 as $N \rightarrow \infty$. Since $q R_{K}^{*}(N)$ is an integer, we infer that $R_{K}^{*}(N)=0$ for $N \geq N_{0}$. It follows that

$$
0=(1+o(1)) N F^{(K)}(N)+O(1) \quad \text { for } N \geq N_{0},
$$

which contradicts $(21)$.

Corollary 3.5. Let $\alpha \in \mathbb{R}_{\geq 0}, \gamma \in \mathbb{R}_{\geq 0}$. If $\sum_{N=1}^{\infty}\left[\gamma N^{\alpha}\right] / N ! \in \mathbb{Q}$, then $\alpha=0$ and $[\gamma]=0$.

Proof. If $\alpha \notin \mathbb{Z}$, then apply Theorem 3.4 with $a=1, b=0, K=[\alpha]$, $f(N)=\left[\gamma N^{\alpha}\right], F(N)=\gamma N^{\alpha-1}$. If $\alpha \in \mathbb{Z}$, then apply Corollary 3.4 with $T=\alpha$.

REMARK. It is remarkable that Corollary 3.5 holds for all $\alpha \geq 0$ and $\gamma>0$ so that $\sum_{N=1}^{\infty}\left[\gamma N^{\alpha}\right] / N$ ! is a strictly monotonic function of $\alpha$ and $\gamma$ missing all rational values.

Corollary 3.6. Let $\alpha \in \mathbb{R}_{\geq 0} \backslash \mathbb{Z}, \gamma \in \mathbb{R}_{+}$. Then

$$
\sum_{N=1}^{\infty}\left[\gamma N^{\alpha} \log N\right] / N ! \notin \mathbb{Q} .
$$

Proof. Apply Theorem 3.4 with $a=1, b=0, K=[\alpha], f(N)=$ $\left[\gamma N^{\alpha} \log N\right], F(N)=\gamma N^{\alpha-1} \log N$.

Theorem 3.4 is not strong enough to prove that $\sum_{N=1}^{\infty}[N \log N] / N$ ! is irrational. For such series we provide a variant of Theorem 3.4 where condition (21) becomes weaker, but conditions (18) and (19) become stronger.

Theorem 3.5. Let $K \geq 1, a>0$ and $b$ be given integers such that an $+b \neq 0$ for every $n \in \mathbb{N}$. Let $F: \mathbb{R}_{+} \rightarrow \mathbb{R}_{+}$be a function such that

$$
\begin{gathered}
F(N+x)=\sum_{r=0}^{\infty} \frac{F^{(r)}(N)}{r !} x^{r} \quad \text { for } x=o(N) \text { as } N \rightarrow \infty, \\
F^{(r)}(N)=O\left(r ! \frac{F(N)}{N^{r}}\right) \quad \text { uniformly for } r=0,1, \ldots \text { as } N \rightarrow \infty,
\end{gathered}
$$




$$
\begin{gathered}
\lim _{x \rightarrow \infty} F^{(K)}(x)=0, \quad \lim _{x \rightarrow \infty} \frac{x^{K+1}\left|F^{(K)}(x)\right|}{F(x)}=\infty, \\
\lim _{x \rightarrow \infty} x^{2}\left|F^{(K)}(x)\right|=\infty .
\end{gathered}
$$

Let $f: \mathbb{N} \rightarrow \mathbb{Z}$ be a sequence such that

$$
R^{*}:=\sum_{N=1}^{\infty} \frac{f(N)}{\prod_{n=1}^{N}(a n+b)}
$$

is absolutely convergent and $f(N)=(a N+b) F(N)+O(1)$ as $N \rightarrow \infty$. Then $R^{*}$ is irrational.

Proof. Let $N$ be sufficiently large. Suppose $R^{*}=p / q$ where $p$ and $q>0$ are coprime integers. Conditions (22) and (23) imply conditions (18) and (19). Hence, by Theorem 3.4, we may assume without loss of generality that

$$
N F^{(K)}(N)=O(1) \text {. }
$$

Observe that by (23) and (22), for $x=o(N)$,

$$
F(N+x) \leq \sum_{r=0}^{\infty}\left|F^{(r)}(N)\right| \frac{x^{r}}{r !}=O\left(F(N) \sum_{r=0}^{\infty}\left(\frac{x}{N}\right)^{r}\right)=O(F(N)) .
$$

Put

$$
R_{K-1}^{*}(N)=\sum_{j=0}^{K-1}(-1)^{j}\left(\begin{array}{c}
K-1 \\
j
\end{array}\right) \sum_{s=0}^{\infty} \frac{f(N+j+s)}{\prod_{n=N+j}^{N+j+s}(a n+b)} .
$$

By Lemma 2.1, $q R_{K-1}^{*}(N)$ is an integer for every positive integer $N$. We have, by Lemma 2.5 ,

$$
\begin{aligned}
R_{K-1}^{*}(N) & =\sum_{j=0}^{K-1}(-1)^{j}\left(\begin{array}{c}
K-1 \\
j
\end{array}\right) \sum_{s=0}^{\infty} \frac{F(N+j+s)}{\prod_{n=N+j}^{N+j+s-1}(a n+b)}+O\left(\frac{1}{N}\right) \\
& =(-1)^{K-1} F^{(K-1)}(N)+O\left(\frac{F(N)}{N^{K}}+\frac{1}{N}\right) .
\end{aligned}
$$

Put

$$
\begin{aligned}
& t=1+\left[\frac{N}{\min _{x \in[N, 2 N]}\left(x^{2}\left|F^{(K)}(x)\right|\right)^{1 / 2}}\right. \\
& \left.+\frac{N}{\min _{x \in[N, 2 N]}\left(x^{K+1}\left|F^{(K)}(x)\right| / F(x)\right)^{1 / 2}}\right] .
\end{aligned}
$$

It follows from (25) and (24) that $t=o(N)$ as $N \rightarrow \infty$. Hence, by (27), similarly to (28),

$$
q R_{K-1}^{*}(N+t)=(-1)^{K-1} q F^{(K-1)}(N+t)+O\left(\frac{F(N)}{N^{K}}+\frac{1}{N}\right)
$$

is an integer. 
We apply the Mean Value Theorem to (30) and (28). Hence there exists a real number $\tau$ with $0<\tau<t$ such that

$$
\begin{aligned}
M(N) & :=q R_{K-1}^{*}(N+t)-q R_{K-1}^{*}(N) \\
& =(-1)^{K-1} q t F^{(K)}(N+\tau)+O\left(\frac{F(N)}{N^{K}}+\frac{1}{N}\right)
\end{aligned}
$$

is an integer. It follows from (29), (27) and (24) that, for some positive constant $c$,

$$
\begin{aligned}
\frac{t N^{K}\left|F^{(K)}(N+\tau)\right|}{F(N)} & \geq \frac{N^{K+1}\left|F^{(K)}(N+\tau)\right|}{F(N) \min _{x \in[N, 2 N]}\left(x^{K+1}\left|F^{(K)}(x)\right| / F(x)\right)^{1 / 2}} \\
& \geq c\left(\frac{(N+\tau)^{K+1}\left|F^{(K)}(N+\tau)\right|}{F(N+\tau)}\right)^{1 / 2} \rightarrow \infty \quad \text { as } N \rightarrow \infty .
\end{aligned}
$$

Hence, by (26) and $t=o(N)$,

$$
M(N)=(-1)^{K-1}(1+o(1)) q t F^{(K)}(N+\tau)+O(1 / N) \rightarrow 0 .
$$

Thus, since $M(N)$ represents an integer, $M(N)=0$ for $N \geq N_{1}$. It follows from (31), (29) and (27) that

$$
\begin{aligned}
0 & =N t\left|F^{(K)}(N+\tau)\right|+O(1) \\
& \geq \frac{N^{2}\left|F^{(K)}(N+\tau)\right|}{\min _{x \in[N, 2 N]}\left(x^{2}\left|F^{(K)}(x)\right|\right)^{1 / 2}}+O(1) \\
& \geq\left((N+\tau)^{2}\left|F^{(K)}(N+\tau)\right|\right)^{1 / 2}+O(1),
\end{aligned}
$$

which contradicts (25).

Corollary 3.7. Let $\alpha \in \mathbb{R}_{\geq 0}, \beta \in \mathbb{R}, \beta \neq 0, \gamma \in \mathbb{R}_{+}$. Suppose $\beta>0$ whenever $\alpha=0$. Then

$$
\sum_{N=1}^{\infty} \frac{\left[\gamma N^{\alpha} \log ^{\beta} N\right]}{N !} \notin \mathbb{Q} .
$$

Proof. Apply Theorem 3.5 with $a=1, b=0, K=[\alpha]$ if $\alpha \notin \mathbb{Z}$ or $\beta>0$, and $K=\alpha-1$ otherwise, $f(N)=\left[\gamma N^{\alpha} \log ^{\beta} N\right], F(N)=\gamma N^{\alpha-1} \log ^{\beta} N$.

Corollary 3.8. Let $\alpha \in \mathbb{R}_{\geq 0}, 0<\beta<1, \gamma \in \mathbb{R}_{+}$. Then

$$
\sum_{N=1}^{\infty} \frac{\left[\gamma N^{\alpha} \exp \left(\log ^{\beta} N\right)\right]}{N !} \notin \mathbb{Q} .
$$

Proof. Apply Theorem 3.5 with $a=1, b=0, K=[\alpha], f(N)=$ $\left[\gamma N^{\alpha} \exp \left(\log ^{\beta} N\right)\right], F(N)=\gamma N^{\alpha-1} \exp \left(\log ^{\beta} N\right)$. 
4. Linear independence. The method from the previous section enables us to prove the linear independence of the considered sums. Recall that Theorem 3.1 precisely states when

$$
\sum_{N=1}^{\infty} \frac{P(N)}{\prod_{n=1}^{N}(a n+b)}
$$

for $P(x) \in \mathbb{Z}[x]$ is rational.

THEOREM 4.1. Let $a>0$ and $b$ be integers such that $a n+b \neq 0$ for every $n \in \mathbb{N}$. Suppose that $P(x)=\sum_{i=0}^{T} a_{i} x^{i} \in \mathbb{Z}[x]$ and that

$$
\sum_{N=1}^{\infty} \frac{P(N)}{\prod_{n=1}^{N}(a n+b)}
$$

is irrational. Let $W$ be a set of functions $F: \mathbb{R}_{+} \rightarrow \mathbb{R}_{+}$which have the following properties:

(i)

$$
F(N+x)=\sum_{r=0}^{\infty} \frac{F^{(r)}(N)}{r !} x^{r} \quad \text { for } x=o(N) \text { as } N \rightarrow \infty
$$

(ii)

(iii) either there exists a positive integer $K$ such that

$$
F^{(K)}(x)=o(1), \quad \frac{F(x)}{x^{K+1}}=o\left(\left|F^{(K)}(x)\right|\right), \quad \lim _{x \rightarrow \infty} x^{2}\left|F^{(K)}(x)\right|=\infty
$$

or

$$
K=0, \quad \lim _{x \rightarrow \infty} F(x)=0, \quad \lim _{x \rightarrow \infty} x F(x)=\infty,
$$

(iv) for every pair of functions $F, G \in W$ with corresponding integers $K>L$ one has $\lim _{x \rightarrow \infty} G^{(k)}(x) / F^{(k)}(x)=0$ for $k=0,1, \ldots, K$; for every pair of functions $F, G \in W$ with $F \neq G$ and corresponding integers $K=L$ one has either $\lim _{x \rightarrow \infty} G^{(k)}(x) / F^{(k)}(x)=0$ for $k=0,1, \ldots, K$ or $\lim _{x \rightarrow \infty} F^{(k)}(x) / G^{(k)}(x)=0$ for $k=0,1, \ldots, K$.

Suppose that for every function $F \in W$ there exists a function $f: \mathbb{N} \rightarrow \mathbb{Z}$ such that

$$
\sum_{N=1}^{\infty} \frac{f(N)}{\prod_{n=1}^{N}(a n+b)}
$$

is absolutely convergent and

$$
f(N)=(a N+b) F(N)+O(1) \quad \text { as } N \rightarrow \infty .
$$


Then the numbers

$$
\sum_{N=1}^{\infty} \frac{f(N)}{\prod_{n=1}^{N}(a n+b)} \quad(F \in W), \quad \sum_{N=1}^{\infty} \frac{P(N)}{\prod_{n=1}^{N}(a n+b)}, \quad 1
$$

are linearly independent over the rationals.

Proof. Suppose that there exist functions $f_{0}:=P, f_{1}, f_{2}, \ldots, f_{M} \in W$ such that the numbers

$$
\sum_{N=1}^{\infty} \frac{f_{i}(N)}{\prod_{n=1}^{N}(a n+b)}
$$

$(i=0,1, \ldots, M)$ and the number 1 are linearly dependent over the rationals. Then there exist integers $A_{0}, A_{1}, \ldots, A_{M}, p$ and $q>0$, not all zero, such that

$$
\frac{p}{q}=\sum_{m=0}^{M} A_{m} \sum_{N=1}^{\infty} \frac{f_{m}(N)}{\prod_{n=1}^{N}(a n+b)}
$$

It is excluded that $A_{1}=\cdots=A_{M}=0$. Without loss of generality we may assume that $A_{1}, \ldots, A_{M}$ are all nonzero and $M>0$. Lemma 3.1 and equation (36) imply

$$
\begin{aligned}
\frac{p}{q} & =A_{0} \sum_{N=1}^{\infty} \frac{P(N)}{\prod_{n=1}^{N}(a n+b)}+\sum_{m=1}^{M} A_{m} \sum_{N=1}^{\infty} \frac{f_{m}(N)}{\prod_{n=1}^{N}(a n+b)} \\
& =\frac{p_{1}}{q_{1}}+\frac{p_{2}}{q_{2}} \sum_{N=1}^{\infty} \frac{1}{\prod_{n=1}^{N}(a n+b)}+\sum_{N=1}^{\infty} \frac{\sum_{m=1}^{M} A_{m} f_{m}(N)}{\prod_{n=1}^{N}(a n+b)}
\end{aligned}
$$

where $p_{1}, p_{2}, q_{1}>0$ and $q_{2}>0$ are suitable integers which do not depend on $N$. Thus

$$
A:=q_{1} q_{2} q\left(\frac{p}{q}-\frac{p_{1}}{q_{1}}\right)=q_{1} q \sum_{N=1}^{\infty} \frac{p_{2}+q_{2} \sum_{m=1}^{M} A_{m} f_{m}(N)}{\prod_{n=1}^{N}(a n+b)}
$$

is an integer.

By Lemma 2.1, for every positive integer $N$ the number

$$
S_{N}:=q_{1} q \sum_{s=0}^{\infty} \frac{p_{2}+q_{2} \sum_{m=1}^{M} A_{m} f_{m}(N+s)}{\prod_{n=N}^{N+s}(a n+b)}
$$

is an integer. Moreover

$$
S_{N}=q_{1} q_{2} q \sum_{s=0}^{\infty} \frac{\sum_{m=1}^{M} A_{m} F_{m}(N+s)}{\prod_{n=N}^{N+s-1}(a n+b)}+O\left(\frac{1}{N}\right)
$$


Without loss of generality we may assume by (iv) and (iii) that if $K$ is the integer corresponding to $F_{M}$, then

(38) $\lim _{x \rightarrow \infty} \frac{F_{m}^{(k)}(x)}{F_{M}^{(k)}(x)}=0 \quad$ for $k=0,1, \ldots, K$ and $m=0,1, \ldots, M-1$.

Let $L$ be a nonnegative integer with $L \leq K$. We deduce from (37) that

$$
\begin{aligned}
R_{L}^{*}(N) & :=\sum_{j=0}^{L}(-1)^{j}\left(\begin{array}{c}
L \\
j
\end{array}\right) S_{N+j} \\
& =q_{1} q_{2} q \sum_{j=0}^{L}(-1)^{j}\left(\begin{array}{c}
L \\
j
\end{array}\right) \sum_{s=0}^{\infty}\left(\frac{\sum_{m=1}^{M} A_{m} F_{m}(N+s+j)}{\prod_{n=N}^{N+s+j-1}(a n+b)}\right)+O\left(\frac{1}{N}\right)
\end{aligned}
$$

is an integer as well. From Lemma 2.5, (32), and (33) we deduce that

$$
R_{L}^{*}(N)=(-1)^{L} q_{1} q_{2} q \sum_{m=1}^{M} A_{m} F_{m}^{(L)}(N)+\sum_{m=1}^{M} O\left(F_{m}(N) N^{-L-1}\right)+O\left(\frac{1}{N}\right) .
$$

Hence, by (38),

$$
R_{L}^{*}(N)=(-1)^{L} q_{1} q_{2} q \sum_{m=1}^{M} A_{m} F_{m}^{(L)}(N)+O\left(F_{M}(N) N^{-L-1}\right)+O(1 / N) .
$$

We distinguish two cases.

(a) Suppose $\lim \sup _{N \rightarrow \infty} N\left|F_{M}^{(K)}(N)\right|=\infty$. Then we put $L=K$ and find by (38) that

$$
R_{K}^{*}(N)=(-1)^{K} q_{1} q_{2} q A_{M} F_{M}^{(K)}(N)(1+o(1))+O\left(F_{M}(N) N^{-K-1}\right)+O\left(\frac{1}{N}\right) .
$$

Hence we derive a contradiction in the same way as we did in the last lines of the proof of Theorem 3.4.

(b) Suppose $\lim \sup _{N \rightarrow \infty} N\left|F_{M}^{(K)}(N)\right|<\infty$, i.e. $F_{M}^{(K)}(N)=O(1 / N)$ as $N \rightarrow \infty$. Note that $K \geq 1$ in view of (35). By (27) and (38) we see, for $m=1, \ldots, M$ and $x=o(N)$, that $F_{m}(N+x)=O\left(F_{m}(N)\right)=O\left(F_{M}(N)\right)$. Put $L=K-1$ in (39). Hence

$$
R_{K-1}^{*}(N)=(-1)^{K-1} q_{1} q_{2} q \sum_{m=1}^{M} A_{m} F_{m}^{(K-1)}(N)+O\left(F_{M}(N) N^{-K}\right)+O\left(\frac{1}{N}\right) .
$$

Define $t$ as in (29). We apply the Mean Value Theorem to the integer $M(N):=R_{K-1}^{*}(N+t)-R_{K}^{*}(N)$. We obtain

$$
M(N)=(-1)^{K-1} q_{1} q_{2} q t \sum_{m=1}^{M} A_{m} F_{m}^{(K)}(N+\tau)+O\left(F_{M}(N) N^{-K}\right)+O\left(\frac{1}{N}\right)
$$


for some $\tau$ with $0<\tau<t$. By (38) we have

$$
M(N)=(-1)^{K-1} q_{1} q_{2} q t(1+o(1)) A_{M} F_{M}^{(K)}(N+\tau)+O\left(\frac{F_{M}(N)}{N^{K}}+\frac{1}{N}\right) .
$$

The further proof proceeds as that of Theorem 3.5 from the introduction of $M(N)$ on.

REMARK. It follows from a repeated use of de l'Hôpital's rule that condition (iv) can be relaxed. If

$$
\lim _{x \rightarrow \infty} \frac{F^{(K)}(x)}{G^{(K)}(x)}=0 \quad \text { and } \quad \lim _{x \rightarrow \infty} G^{(K-1)}(x)=\infty,
$$

then $\lim _{x \rightarrow \infty} F^{(k)}(x) / G^{(k)}(x)=0$ for $k=0,1, \ldots, K$. (See Theorem 5.13 in [13], with $A=0$ and $a=\infty$.)

Corollary 4.1. The numbers 1 , e and $\sum_{n=1}^{\infty}\left[n^{\alpha}\right] / n !\left(\alpha \in \mathbb{R}_{+}, \alpha \notin \mathbb{Z}\right)$ are linearly independent over the rationals.

Corollary 4.2. Let $\alpha_{1}, \ldots, \alpha_{M}$ be positive real numbers and let $P_{1}, \ldots, P_{M}$ be nonzero polynomials with integer coefficients such that the numbers $\alpha_{m} \operatorname{deg} P_{m}$ are distinct and nonintegral. Then the numbers $1, e$ and $\sum_{N=1}^{\infty}\left[N^{\alpha_{m}} P_{m}(N)\right] / N !(m=1, \ldots, M)$ are linearly independent over the rationals.

Proof. Apply Theorem 4.1 with $P(x)=1$ and $F_{m}(X)=x^{\alpha_{m}-1} P_{m}(x)$ for $m=1, \ldots, M$.

Corollary 4.3. The numbers 1 and $\sum_{n=1}^{\infty}\left[n(\log n)^{\alpha}\right] / n ! \quad(\alpha \in \mathbb{R})$ are linearly independent over the rationals.

Remark. Conditions (i), (ii) and (iii) of Theorem 4.1 are satisfied by a large class of functions comprising

$$
\begin{aligned}
\gamma x^{\alpha} & \left(\alpha>-1, \alpha \notin \mathbb{Z}, \gamma \in \mathbb{R}_{+}\right) \text {with } K=[\alpha]+1, \\
\gamma e^{\beta(\log x)^{\alpha}} & \left(0<\alpha<1, \beta \in \mathbb{R}_{+}, \gamma \in \mathbb{R}_{+}\right) \text {with } K=1, \\
\gamma(\log x)^{\alpha} & \left(\alpha \neq 0, \gamma \in \mathbb{R}_{+}\right) \text {with } K=1 \text { if } \alpha>0, K=0 \text { if } \alpha<0, \\
\gamma(\log \log x)^{\alpha} & \left(\alpha \neq 0, \gamma \in \mathbb{R}_{+}\right) \text {with } K=1 \text { if } \alpha>0, K=0 \text { if } \alpha<0 .
\end{aligned}
$$

It is therefore possible to apply Theorem 4.1 to sums and products of such functions and polynomials provided that condition (iv) is satisfied.

EXAMPLE 4.1. The numbers

$$
\text { 1, } \quad \sum_{n=1}^{\infty} \frac{\left[(\log n)^{1 / 2}\right]}{n !}, \quad \sum_{n=1}^{\infty} \frac{\left[e^{(\log n)^{1 / 2}}\right]}{n !}
$$

are linearly independent over the rationals. 


\section{References}

[1] M. Aigner, Combinatorial Theory, Classics Math., Springer, Berlin, 1997.

[2] G. Cantor, Über die einfachen Zahlensysteme, Z. Math. Phys. 14 (1869), 121-128; Collected Papers, Springer, Berlin, 1932, 35-42.

[3] P. Erdős, Sur certaines séries à valeur irrationnelle, Enseign. Math. (2) 4 (1958), 93-100.

[4] P. Erdős and M. Kac, Problem 4518, Amer. Math. Monthly 61 (1954), 264.

[5] P. Erdös and E. G. Straus, On the irrationality of certain series, Pacific J. Math. 55 (1974), 85-92.

[6] J. Hančl, A note to the rationality of infinite series I, Acta Math. Inform. Univ. Ostraviensis 5 (1997), 5-11.

[7] - A note on a paper of Oppenheim and Šalát concerning the series of Cantor type, ibid. 10 (2002), 35-41.

[8] J. Hančl and R. Tijdeman, On the irrationality of Cantor series, J. Reine Angew. Math. 571 (2004), 145-158.

[9] C. Hermite, Sur la fonction exponentielle, C. R. Acad. Sci. Paris 77 (1873), 18-24, 74-79, 226-233, 285-293; Oeuvres III, Gauthier-Villars, Paris, 1912, 150-181.

[10] J. H. Lambert, Mémoire sur quelques propriétés remarquables des quantités transcendentes circulaires et logarithmiques, Histoire Acad. Roy. Sci. Belles-Lett. Berlin, 1761, 265-322; Opera Mathematica II, Orell Füssli Verlag, Zürich, 1948, 112-159.

[11] J. H. Loxton and A. J. van der Poorten, Arithmetic properties of certain functions in several variables III, Bull. Austral. Math. Soc. 16 (1977), 15-47.

[12] A. Oppenheim, Criteria for irrationality of certain classes of numbers, Amer. Math. Monthly 61 (1954), 235-241.

[13] W. Rudin, Principles of Mathematical Analysis, McGraw-Hill, New York, 1964.

[14] R. Tijdeman and P. Z. Yuan, On the rationality of Cantor and Ahmes series, Indag. Math. (N.S.) 13 (2002), 407-418.

Department of Mathematics

and

Institute for Research

and Applications of Fuzzy Modeling

University of Ostrava

30. dubna 22

70103 Ostrava 1, Czech Republic

E-mail: hancl@osu.cz
Mathematisch Instituut Universiteit Leiden Postbus 9512 Niels Bohrweg 1 2300 RA Leiden, The Netherlands E-mail: tijdeman@math.leidenuniv.nl 